\title{
Improvement of sorghum grain quality using modern genetic tools
}

\author{
L.A. Elkonin ${ }^{1 *}$, V.M. Panin ${ }^{1}$, G.A. Gerashchenkov², O.A. Kenzhegulov ${ }^{1}$ \\ ${ }^{1}$ Agricultural Research Institute of South-East Region, Saratov, Russia \\ ${ }^{2}$ Institute of Biochemistry and Genetics, Subdivision of the Ufa Federal Research Centre, RAS, Ufa, Russia
}

DOI 10.18699/ICG-PlantGen2019-41

(c) Autors, 2019

*e-mail: lelkonin@gmail.com

\begin{abstract}
A review of the studies on the improvement of the nutritional value of sorghum grain by genetic engineering tools is presented. These studies include biofortification of sorghum with amino acids and micronutrients and improving digestibility of seed storage proteins (kafirins) via RNA interference and genome editing. Transgenic sorghum harboring the genetic constructs inducing RNA silencing of kafirins are characterized by an increased in vitro digestibility of endosperm proteins, an increased proportion of lysine, and modification of endosperm texture, which becomes floury devoid of the vitreous layer. In some cases transgenic events with vitreous endosperm and a high digestibility of kafirins were obtained, which may be of interest for practical sorghum breeding. There are evidences that genetic constructs for RNA silencing may be unstable in the plant genome and their expression may be influenced by the environment. Induction of mutations that disrupt the functioning of the kafirin genes using genome editing may be a more effective approach for improving the digestibility of kafirins and increasing the nutritional value of sorghum grain.

Key words: kafirins; endosperm; in vitro protein digestibility; RNAi; CRISPR/Cas9; Sorghum bicolor.
\end{abstract}

\section{Introduction}

One of the promising areas of biotechnology is the improvement of the nutritional value of grain. This task is particularly important for sorghum, a high-yielding drought-tolerant cereal, which is a source of feed and food for millions of people in many countries around the world. However, compared to other cereals, the majority of sorghum cultivars are characterized by a number of nutritional constrains, such as a lower digestibility of seed storage proteins (kafirins) and starch, a poor nutritional value of kafirins, which have a low content of essential amino acids.

Among the factors that cause or may effect this phenomenon are the chemical structure of kafirins, which are abundant with sulphur-containing amino acids capable to form S-S bonds resistant to protease digestion; interactions of kafirins with non-kafirin proteins and non-protein components such as polyphenols and polysaccharides; the spatial organization of different kafirins in the protein bodies of endosperm cells (Belton et al., 2006). It is generally accepted that the peripheral disposition of $\gamma$-kafirin, which is considered as the most stable against protease digestion reduces digestibility of $\alpha$-kafirin, the major sorghum seed storage protein located centrally in protein bodies and comprising up to $80 \%$ of the total endosperm kafirins. In addition, $\gamma$-kafirin is characterized by the ability to form oligo- or polymers of high molecular weight, which are resistant to protease digestion.

In this paper, we summarized some experiments on biofortification of sorghum with amino acids and micronutrients and on improving digestibility of kafirins using genetic engineering approaches and outlined their importance for practical sorghum breeding.

\section{Sorghum biofortification with amino acids and micronutrients}

Numerous researches published up to date clearly demonstrate that genetic engineering techniques are quite promising for enrichment of cereal grain with essential amino acids, i. e. lysine, tryptophan, methionine, and micronutrients. By using endosperm-specific promoters, expression of the desired genes may be provided exactly in the kernels, rather than in somatic tissues. Sorghum grain has a low content of lysine and $\beta$-carotene (provitamin A), which are highly important for human health.

There are reports on obtaining transgenic sorghum plants with an increased content of lysine (up to 40-60\%) (Zhao et al., 2010) and $\beta$-carotene (up to $7.3-12.3 \mu \mathrm{g} / \mathrm{g}$ vs. $0.5 \mu \mathrm{g} / \mathrm{g}$ in non-transgenic control seeds). In order to increase $\beta$-carotene content in sorghum grain and ensure its stability during grain storage, the introduction of five genetic constructs encoding enzymes involved in the carotenoid biosynthesis pathway and preventing its oxidative degradation was performed (Che et al., 2016).

This research is a shining example of the effectiveness of the genetic engineering approach for modifying plant metabolism to meet human needs.

\section{Increase in seed storage protein digestibility using RNA-silencing technology}

In recent years, RNA interference technology has been intensively used to suppress the synthesis of seed storage proteins in different species of cereals including wheat, rice and maize. These experiments contributed to obtaining new information on the mechanisms of protein body formation, as well as the 
role of various classes of prolamins and glutenins in the development of endosperm and their effect on the technological properties of flour.

In sorghum, intensive studies on the induction of RNAsilencing of the kafirin genes have been undertaken by several research groups (DaSilva et al., 2011; Kumar et al., 2012; Grootboom et al., 2014). The main goal of these experiments was the improvement of in vitro protein digestibility as a result of suppressing synthesis of different kafirin sub-classes. In these experiments, RNAi-constructs differ in the number of targeted kafirin genes, the nature of intron separated inverted repeats of kafirin genes, and promoters driving these constructs.

In the experiments of each of these groups, lines of transgenic sorghum with suppressed synthesis of $\gamma$ - and/or $\alpha$-kafirins and a floury type of endosperm were obtained. Unfortunately, the presence of floury endosperm is a disadvantage of these lines, since the absence of a vitreous layer increases the fragility of the grain and reduces their resistance to damage by fungal diseases.

Remarkably, in our experiments with genetic constructs for the silencing of only the $\gamma$-kafirin gene (Elkonin et al., 2016) we have obtained transgenic plants, which had kernels with normal vitreous endosperm, as well as with a thin vitreous endosperm layer, with floury or modified endosperm types. In kernels with the modified endosperm type, the vitreous layer was significantly reduced and developed as sectors or patches surrounded by floury endosperm. These kernels resemble the kernels of recombinant sorghum lines obtained by hybridization of a highly digestible mutant with floury endosperm $(h d h l)$ with ordinary sorghum lines with vitreous endosperm (Tesso et al., 2006).

Previously, transgenic plants with patches of vitreous endosperm surrounded by floury endosperm were also observed in 'Tx 430' containing a genetic construct for silencing $\alpha$ - and $\gamma$-kafirins, while co-suppression of $\delta$-kafirin and $\gamma$-kafirin subclasses did not change the endosperm phenotype in this line (DaSilva, 2012). Apparently, the formation of different endosperm types results from peculiarities of expression of the genetic constructs in the genome of the recipient line.

The most important effect of kafirin silencing in transgenic plants was an increased protein digestibility in both cooked and raw sorghum flour. For example, transgenic plants of 'Tx430' carrying the genetic construct for silencing of $\alpha$ and $\gamma$-kafirin sub-classes were characterized by an improved in vitro protein digestibility of both raw and cooked grain flour: 78 and $61 \%$, respectively; while in the non-transgenic control these figures were 40-50 and 34-40\%, respectively (DaSilva et al., 2011).

The genetic construct for silencing only $\delta$ - and $\gamma$-kafirins also caused an improvement of in vitro protein digestibility of raw flour, but did not affect the digestibility of cooked flour (DaSilva, 2012). In experiments by Kumar et al. (2012), cooked flour obtained from transgenic plants carrying genetic constructs for silencing $\gamma$-kafirin did not differ from the nontransgenic control, while the silencing of $\alpha$-kafirin improved the protein digestibility of cooked flour.

Transgenic plants obtained in our experiments also had a significantly improved in vitro digestibility of endosperm pro- teins (Elkonin et al., 2016). A comparison of electrophoretic spectra before and after pepsin digestion revealed that the amount of undigested $\alpha$-kafirin monomers and total undigested protein was significantly lower (1.7-1.9 times, according to quantitative analysis of SDS-PAGE) in the transgenic plant than in the original non-transgenic line.

The digestibility value reached $85.4 \%$, while in original line this value was about $60 \%$. Remarkably, in the kernels of transgenic plant \#94-3-08 ( $\left.\mathrm{T}_{2}\right)$ with thick vitreous endosperm, the differences in kafirin digestion were more pronounced: the amount of undigested monomers was 17.5 as low, and the amount of total undigested protein was 4.7 as low as those in the original line. The digestibility value reached $92 \%$.

Plants from $\mathrm{T}_{3}$ generation inherited the improved digestibility of kafirins. In these plants, kernels had either floury or modified endosperm or endosperm with a vitreous layer. The level of digestibility of endosperm proteins in these plants was $83-90 \%$, significantly differing from the digestibility of proteins in the original non-transgenic line. Apparently, a decrease in $\gamma$-kafirin increases the digestibility of $\alpha$-kafirins. This increase may be due to chemical reasons (reduction of polymerization) and/or physical reasons (change in the spatial arrangement of $\alpha$-kafirins in the protein bodies that increase their availability to pepsin digestion).

The effect of increased protein digestibility was also observed in some plants from $\mathrm{T}_{4}$ generation, while in others it disappeared owing to the instability of the RNAi construct (see section 5).

\section{Rebalancing of the seed proteome and improvement of the nutritional value of sorghum grain by silencing kafirin genes}

An important consequence of silencing of prolamine genes in cereals is the enhancement of the synthesis of other proteins, including those that have a higher content of essential amino acids, lysine and threonine. Maize plants with silenced $\alpha$-zeins were characterized by a doubled content of the essential amino acids tryptophan and lysine (Huang et al., 2006).

In rice, it has been shown that gene silencing of a $13 \mathrm{kDa}$ prolamine increases the total lysine content up to $56 \%$ as a result of a compensatory rise in the synthesis of lysine-rich glutelins, globulins and chaperones (Kawakatsu et al., 2010). A significant increase in lysine content (up to $3.3 \mathrm{~g} / 100 \mathrm{~g}$ protein in comparison with $2.1 \mathrm{~g} / 100 \mathrm{~g}$ protein in the nontransgenic control) was found in transgenic sorghum plants carrying complex genetic constructs for RNAi silencing of the $\alpha-, \gamma-, \delta$-kafirin genes and lysine-ketoglutarate reductase gene, which control the catabolism of free lysine (DaSilva, 2012).

In our studies, in the kernels of transgenic plants from $\mathrm{T}_{2}$ generation with high in vitro protein digestibility, the total amino acid content was significantly reduced to $22.8-40.2 \%$, in comparison with the original non-transgenic line (Elkonin et al., 2016). At the same time, the relative content of two major essential amino acids, lysine and threonine, significantly increased. The lysine proportion increased 1.6-1.7 times: from $1.54 \%$ of the total amino acid content in the flour of the original non-transgenic line to $2.41-2.63 \%$ in the transgenic plants. Such an increase, coupled with a significant reduc- 
tion in the total level of amino acids, was presumably caused by a decrease in the content of $\alpha$-kafirins poor in lysine and threonine, while the synthesis of other proteins remained undisturbed. Consequently, the relative proportions of lysine and threonine increased. Perhaps, the suppression of the $\gamma$-kafirin synthesis prevents the accumulation of $\alpha$-kafirins, but does not affect the synthesis of other proteins richer in lysine and threonine.

Such a rebalancing of the seed proteome is a common phenomenon for transgenic plants with genetic constructs for RNA silencing of major seed storage proteins. For maize, it was suggested that developing kernels possess compensatory mechanisms that sense protein content when zein synthesis is interrupted, leading to translation of other mRNAs instead of zein mRNAs (Wu, Messing, 2014).

Remarkably, in knockdown mutant lines of soybean with suppressed synthesis of major storage proteins, the seeds maintained nearly identical levels of total protein compared to the untransformed soybean cultivars (Schmidt et al., 2011). These data suggest that proteome rebalancing in seeds might be a rather common event, providing a constant sink for reduced nitrogen during seed maturation.

\section{Instability of genetic constructs for RNA silencing of $\gamma$-kafirin gene}

In our experiments, we found that the progeny of transgenic plants with high in vitro protein digestibility sometimes lost this trait. Even different panicles of one and the same plant had different values of in vitro protein digestibility. PCR analysis of plants from the late generations $\left(\mathrm{T}_{4}, \mathrm{~T}_{5}\right)$ showed elimination of the construct for RNA silencing in the course of plant ontogenesis. Nevertheless, in some plants from the same progeny the construct was stable.

In addition, in some plants from $\mathrm{T}_{4}$ generations, we found elimination of the nos-promoter governing the marker bar gene from the transgenic construct for silencing the $\gamma$-kafirin gene. Therefore, these plants, in fact, turned to be functionally marker-free transgenic plants.

\section{Editing of nucleotide sequences of kafirin genes}

In above examples, suppressing the synthesis of kafirins is performed by small interfering RNAs (siRNAs) and subsequent mRNA cleavage and translational inhibition, which are important epigenetic mechanisms in plants. However, epigenetic mechanisms in plants are known to be sensitive to growing conditions and environmental factors (temperature, soil moisture and air, etc.). There are reports that temperature can have a major impact on the extent of gene silencing. Recently it has been shown that miRNA-mediated mRNA cleavage and translational inhibition are dependent on plant growth temperature (von Born et al., 2018). Therefore, the effectiveness of suppression of kafirin synthesis by RNAi constructs might be sensitive to plant growing conditions. In addition, genetic construct for RNA silencing may be unstable in the plant genome. In this regard, induction of mutations that disrupt the functioning of the kafirin genes at the genetic level may be a more effective approach for altering kafirin levels.
Recently, using the CRISPR/Cas9 gene editing tool Li et al. (2018) induced mutations in a conserved region encoding the endoplasmic reticulum signal peptide of $\alpha$-kafirins.

The target site mutations occurring in each $k l C$ gene include small deletions as well as a low frequency of small insertions. $\mathrm{T}_{1}$ and $\mathrm{T}_{2}$ seeds showed reduced $\alpha$-kafirin levels, an altered protein body microstructure, and selected $T_{2}$ events showed a significantly increased grain protein digestibility and lysine content. These data open new prospects for improving the nutritional value of sorghum grain using biotechnological approaches.

\section{References}

Belton P.S., Delgadillo I., Halford N.G., Shewry P.R. Kafirin structure and functionality. J. Cereal Sci. 2006;44:272-286. DOI 10.1016/j. jes.2006.05.004

Che P., Zhao Z.-Y., Glassman K., Dolde D., Hu T.X., Jones T.J., Gruis D.F., Obukosia S., Wambugu F., Albertsen M.C. Elevated vitamin E content improves all-trans beta-carotene accumulation and stability in biofortified sorghum. Proc. Natl. Acad. Sci. USA. 2016;113:11040-11045. DOI 10.1073/pnas.1605689113.

da Silva L.S. Transgenic sorghum: Effects of altered kafirin synthesis on kafirin polymerisation, protein quality, protein body structure and endosperm texture. PhD Thesis. Department of Food Science, Faculty of Natural and Agricultural Sciences, Univ. Pretoria, South Africa. 2012.

da Silva L.S., Taylor J., Taylor J.R. Transgenic sorghum with altered kafirin synthesis: kafirin solubility, polymerization, and protein digestion. J. Agric. Food Chem. 2011;59:9265-9270. DOI 10.1021/ jf201878p.

Elkonin L.A., Italianskaya J.V., Domanina I.V., Selivanov N.Y., Rakitin A.L., Ravin N.V. Transgenic sorghum with improved digestibility of storage proteins obtained by Agrobacterium-mediated transformation. Russ. J. Plant Physiol. 2016;63:678-689. DOI 10.1134/ S1021443716050046.

Grootboom A.W., Mkhonza N.L., Mbambo Z., O’Kennedy M.M., da Silva L.S., Taylor J., Taylor J.R.N., Chikwamba R., Mehlo L. Cosuppression of synthesis of major $\alpha$-kafirin sub-class together with $\gamma$-kafirin-1 and $\gamma$-kafirin-2 required for substantially improved protein digestibility in transgenic sorghum. Plant Cell Rep. 2014;33: 521-537. DOI 10.1007/s00299-013-1556-5.

Huang S., Frizzi A., Florida C.A., Kruger D.E., Luethy M.H. High lysine and high tryptophan transgenic maize resulting from the reduction of both 19- and 22-kD $\alpha$-zeins. Plant Mol. Biol. 2006;61:525535. DOI 10.1007/s11103-006-0027-6.

Kawakatsu T., Hirose S., Yasuda H., Takaiwa F. Reducing rice seed storage protein accumulation leads to changes in nutrient quality and storage organelle formation. Plant Physiol. 2010;154:1842-1854. DOI 10.1104/pp.110.164343.

Kumar T., Dweikat I., Sato S., Ge Z., Nersesian N., Chen H., Elthon T., Bean S., Ioerger B.P., Tilley M., Clemente T. Modulation of kernel storage proteins in grain sorghum (Sorghum bicolor (L.) Moench). Plant Biotechnol. J. 2012;10:533-544. DOI 10.1111/j.14677652.2012.00685.x.

Li A., Jia S., Yobi A., Ge Z., Sato S.J., Zhang C., Angelovici R., Clemente T.E., Holding D.R. Editing of an alpha-kafirin gene family increases digestibility and protein quality in sorghum. Plant Physiol. 2018;177(4):1425-1438. DOI 10.1104/pp.18.00200.

Schmidt M.A., Barbazuk W.B., Sandford M., May G., Song Z., Zhou W., Nikolau B.J., Herman E.M. Silencing of soybean seed storage proteins results in a rebalanced protein composition preserv- 
ing seed protein content without major collateral changes in the metabolome and transcriptome. Plant Physiol. 2011;156:330-345. DOI 10.1104/pp.111.173807.

Tesso T., Ejeta G., Chandrashekar A., Huang C.-P., Tandjung A., Lewamy M., Axtell J.D., Hamaker B.R. A novel modified endosperm texture in a mutant high-protein digestibility/high-lysine grain sorghum (Sorghum bicolor (L.) Moench). Cereal Chem. 2006;83:194-201. DOI 10.1094/CC-83-0194.

von Born P., Bernardo-Faura M., Rubio-Somoza I. An artificial miRNA system reveals that relative contribution of translational inhibition to miRNA-mediated regulation depends on environmental and developmental factors in Arabidopsis thaliana. PLoS ONE. 2018; 13(2):e0192984. DOI 10.1371/journal.pone.0192984
Wu Y., Messing J. Proteome balancing of the maize seed for higher nutritional value. Front. Plant Sci. 2014;5:240. DOI 10.3389/fpls. 2014.00240

Zhao Z.-Y., Glassman K., Sewalt V., Wang N., Miller M., Chang S., Thompson T., Catron S., Wu E., Bidney D., Kedebe Y., Jung R. Nutritionally improved transgenic sorghum. Plant Biotechnol. 2002 and Beyond. Vasil I.K. (Ed.). Springer, Dordrecht, 2003;413-416. DOI 10.1007/978-94-017-2679-5_85.

Acknowledgements. The work was partially supported by Russian Foundation for Basic Researches, grant no.19-016-00117.

Conflict of interest. The authors declare no conflict of interest. 